\title{
Using curve-fitting optimisation technique to estimate power MOSFET model parameters for PECT II system
}

\begin{abstract}
This paper presents a proposed new structure of the power MOSFET model and its implementation in the HSPICE and PECT II (Power Electronics and Control Tool) packages. A fast and accurate technique for determining power MOSFET model parameters which introduces a curve-fitting optimisation technique is discussed in detail. The optimisation process generates automatically a set of device parameters based on the input specification, measured data and manufacturer's data sheets. The input procedure involves a netlist specifying parameter tolerances, component voltage and current limits and an initial guess to the selected parameter. Using curve fitting and iterative methods, the "correct" model is generated for utilisation in the simulation phase of both the HSPICE and PECT II packages. This method is then compared to that of a normal method which uses specific formulae to obtain power MOSFET model parameters. The results show that the curve-fitting optimisation technique produces more accurate power MOSFET model parameters than the other method. Both simulation and experimental results of the power MOSFET characteristics are also included in the discussion.
\end{abstract}

Keyword: MOSFET model parameters; Curve fitting optimization technique; PECT II system 